\title{
Topology Optimization Design of Bus Body Structure Based on Altair-Optistruct

\author{
Xiu-Chun WU ${ }^{1,2, a}$, Wen-Qiang ZHENG ${ }^{1, b,}{ }^{\star}$, Pan ZHOU ${ }^{1, c}$
}

${ }^{1}$ School of Mechanical Engineering, University of Shanghai for Science and Technology, Shanghai 200093, China

${ }^{2}$ School of Automobile, Shanghai Dianji University, Shanghai 200245, China

a58777863@qq.com, bzwenqiang@126.com, '1076018760@qq.com

${ }^{*}$ Corresponding author

Keywords: Bus Body, Finite Element, Topology Optimization, Lightweight.

\begin{abstract}
In this paper, the Finite Element Method (FEM) is used to study the topology optimization of bus body structure by Altair-OptiStruct software. Firstly, based on the maximum principle of optimization space, the topology optimization area is established. Then the topology optimization is calculated under the bending condition and bending and torsion condition. Finally, the performance of the body structure is verified. Results show that the performance of structure has been improved and the weight of the body has decreased by $3.8 \%$ after topology optimized, which can provide reference for the bus body lightweight design.
\end{abstract}

\section{Introduction}

In the initial design of product development, there is no sample structure for reference if you want to carry out a new or improved design, and lack of the successful experience about product. So it is difficult to build model quickly [1]. With the rapid development of FEM and computer technology, the application of structural topology optimization for product design has become more and more important method.

Topology optimization method is a finite element based structural optimization process, increasingly used by engineers to support the development of minimum weight structure by helping to determine the most efficient way to carry a given set of loads using a minimum amount of material and designing within a predefined design space[2].The basic idea of topology optimization is to turn seeking the optimal structure of the topological problem into problem of seeking the optimal material distribution within a given design area. Engineers can establish a relatively effective finite element model through defining loads and constraints [3].

\section{Finite Element Modeling}

Firstly, the component area for optimal design is determined when doing topology optimization. The principle of selection is to cover comprehensively as possible and take into account the practical connection of body frame. In order to decrease the weight of the whole model, some less important parts could be removed [4]. According to the spatial structure of bus body frame, the geometry model of the finite element analysis is determined based on shape, geometry size, boundary conditions and other characteristics of the study object [5], and then the locations of windshield, roof vents and air condition should be reserved. Finally, the optimization design space area of the body frame is established.

Here we mainly study on the topology of the region, so the element size of components and parts must be small. For the mesh size, the smaller the mesh is divided, the higher the precision. But the amount of calculation will be bigger and require substantial computational resources. Therefore, comprehensive consideration is needed when meshing.

The bus body frame is formed by ceiling, chassis, front wall, rear wall and side wall etc. The main study includes ceiling and side wall in this paper. Considering the complexity of the frame, the element size is $30 \mathrm{~mm}$, combining with triangular element and quadrilateral element, and the thickness of the size is $40 \mathrm{~mm}$ in the optimization area. 
The finite element model of the bus frame structure contains of 130136 nodes, 7682 triangular elements and 12800 quadrilateral elements after meshing. The material is $16 \mathrm{Mn}$. Elastic modulus is . Density is and Poisson's ratio is 0.3 . The finite element model of body frame after meshing is shown in Fig. 1.

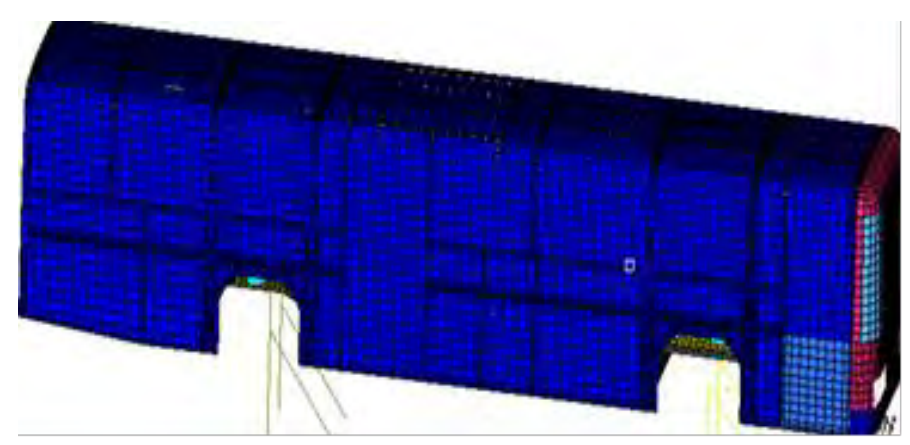

Fig. 1 Topology Optimization Finite Element Model

\section{Topology Optimization}

For a bus, suffered loads are complex and changeable during using. One of the most common conditions mainly includes bending condition and bending and torsion condition in the process of driving, which also is currently the research direction in domestic [6, 7].

Bending condition is the most common form of the bus driving, and the load is received by vertical bending load in this case. The loads are mainly comprised of frame, engine, transmission, seats, luggage etc. The frame weight is loaded in the form of gravity acceleration, and the weight of other parts is loaded with equivalent force, such as seats, passenger and luggage. The weight of the engine and transmission is loaded with corresponding connection of nodes. When applying constraints, $X, Y$ and $Z$ translation DOF of left rear wheel are constrained, $X$ and $Z$ translation DOF of right rear wheel are constrained, $Y$ and $Z$ translation DOF of left front wheel are constrained, and $Z$ translation DOF of right front wheel is constrained. The Load and constraint model of the bending condition is shown in Fig. 2.

Bending and torsion condition is extremely dangerous when the bus driving. It represents the bus's ability to cope with the complex conditions. In this condition, the force of the whole body is considered static. The condition of left front wheel in a suspended state is chosen. The load of this condition is the same with bending condition. When applying constraints, $X, Y$ and $Z$ translation DOF of left rear wheel are constrained, $X$ and $Z$ translation DOF of right rear wheel are constrained, $Y$ and $Z$ translation DOF of left front wheel are constrained, and all DOF of right front wheel are released. The Load and constraint model of bending and torsion condition is shown in Fig. 3.

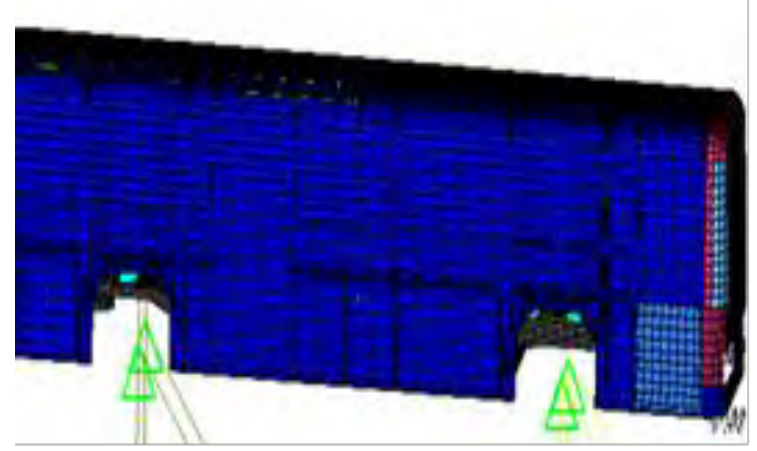

Fig. 2 Applied Load and Constraints Model

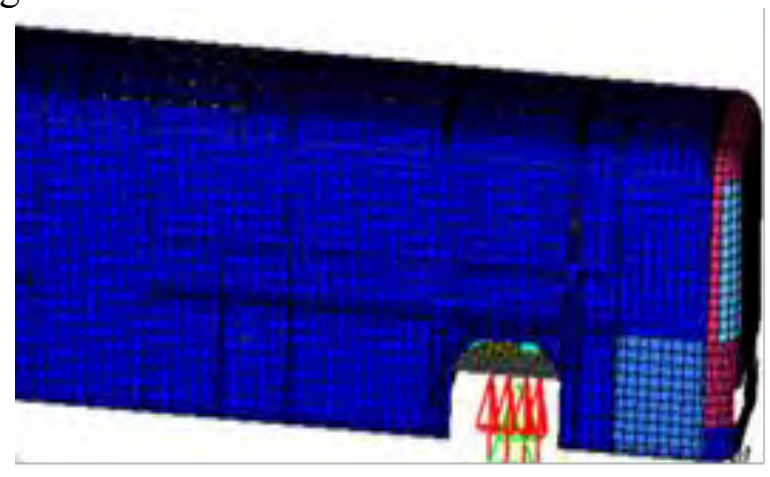

Fig. 3 Applied Load and Constraints Model

The topology optimization design variable is the relative density of optimized space unit. The optimization objective function is the smallest body frame mass. The constraints are bending stiffness and torsion stiffness. Then those are submitted to Altair-OptiStruct. Optimization results are obtained after 60 iterations. The local topological results are shown in Fig. 4 and Fig. 5. 


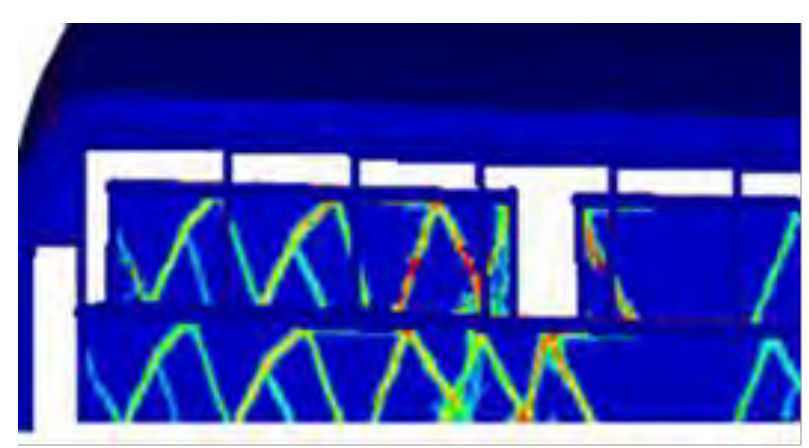

Fig. 4 Optimized Results Nephogram

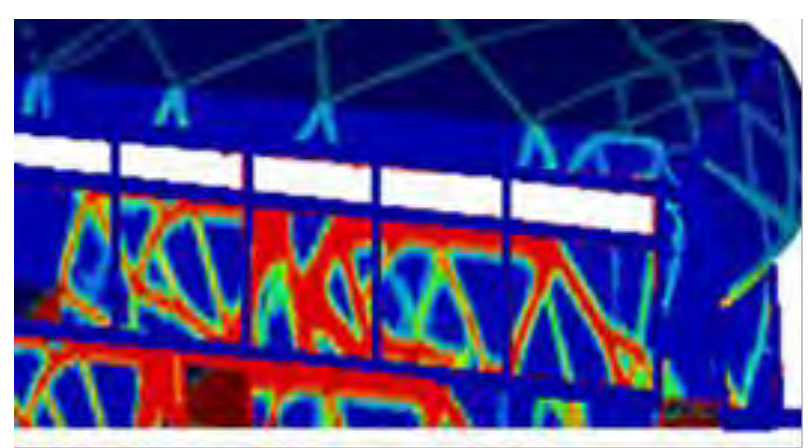

Fig. 5 Optimized Results Nephogram

By comparing the nephogram of two kinds of conditions it can be concluded that topology optimization results are almost the same on the left side wall and right side wall, which indicates that topology optimization results have certain credibility. The load path is more noticeable, and the left and right are surrounded with several obvious beams, and most of them are connected with pillar of the skirt to more convenient to the force transfer and avoid stress concentration. In the bending and torsion condition, the body ceiling consists of a number of triangle structure beams with good stability. The entire topological results are clear and the force transfer path is obvious and reasonable, which shows that simulation analysis is effective. However, some local regions have not reasonable structure, for example, although some places retain the material, but not connect with the surrounding material, so that the force can't be transmitted effectively.

\section{Results Analysis and Performance Comparison}

\section{Optimization Results Analysis}

The body frame component nephogram is obtained by analysis. The nephogram distribution trend and prototype frame as reference, the new body frame is gotten.

The optimization result nephogram of body ceiling is shown in Fig. 6. When processing the results, the beams of triangle structure are chosen as the straight rods. Considering the coordination and load transfer, the junction of the ceiling and side wall should be referred to the structure of the prototype. The optimized structure is shown in Fig. 7.

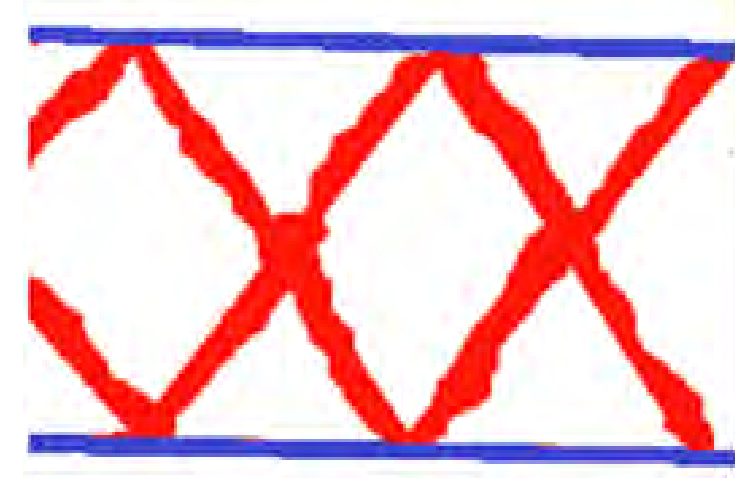

Fig. 6 Ceiling Optimized Results

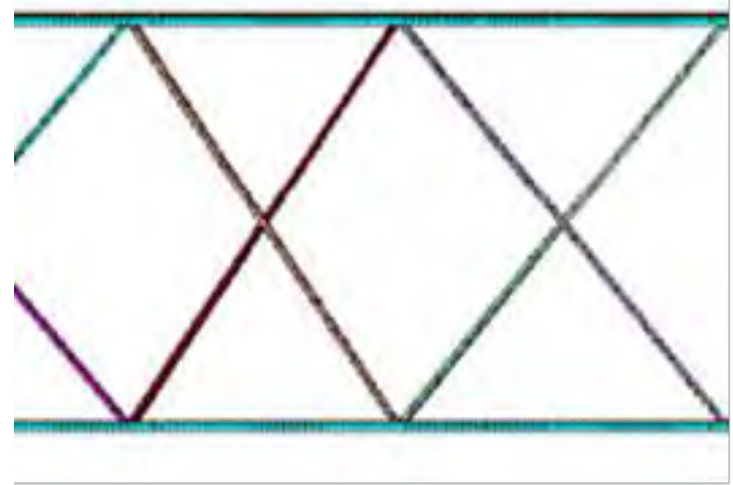

Fig. 7 New Ceiling Structure

The optimization result nephogram of side wall is shown in Fig. 8. The results show that it is beneficial to transfer the load force and avoid stress concentration. Considering the optimization calculation is strictly accordance with the theory, so the components are connected to each other as much as possible during analyzing in order to form a closed structure. According to the load transmission, the beams should be added appropriately. Some short, horizontal or vertical component beams for the connection should be to retain according to the need. In addition, the integral aesthetics and installation convenience of some accessory should be considered. The structure optimization result is shown in Fig. 9. 


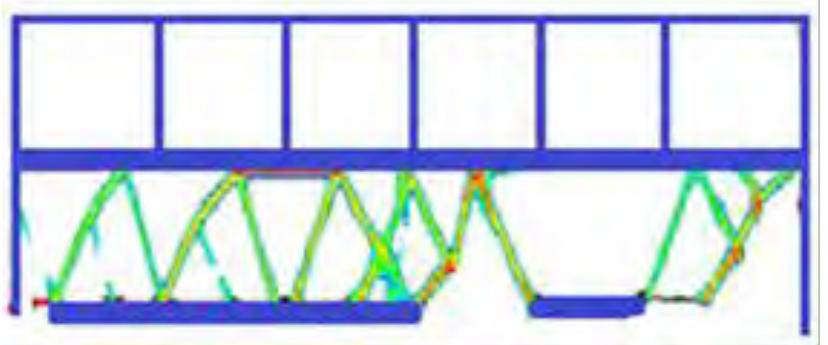

Fig. 8 Side Wall Optimized Results

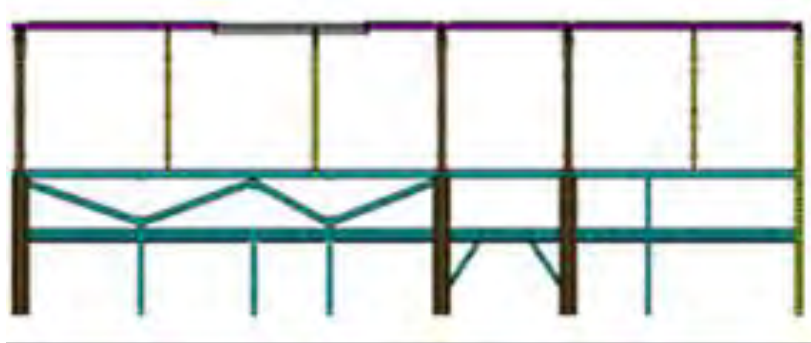

Fig. 9 New Side Wall Structure

\section{Performance Comparison}

After the completion of the relevant analysis and processing, in order to ensure the relevant performance of the frame in the reasonable scope, the static and dynamic characteristics could be analyzed. This paper is mainly analysis and comparison the stiffness and modal characteristics of the frame.

\section{Bending Condition Stiffness Characteristics}

Analysis results chart of the primary position is shown in Fig. 10.

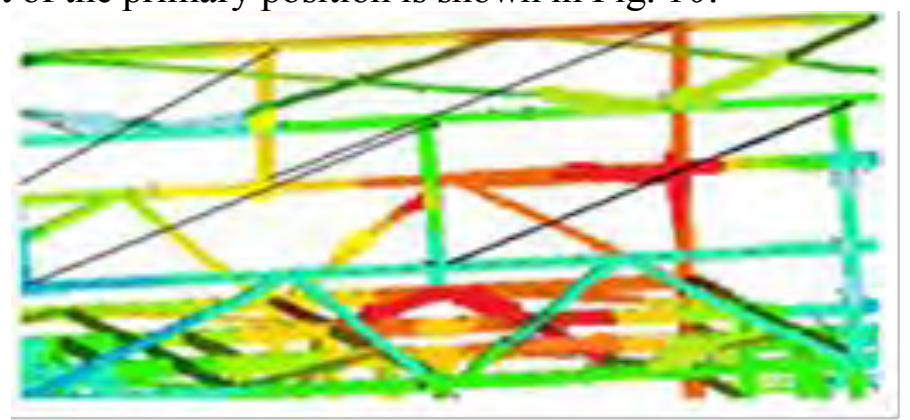

Fig. 10 Bending Condition Measure Diagram

The analysis results show that the largest deformation position of the side walls is on the right side near the position of the door, and the deformation value is $1.791 \mathrm{~mm}$, which indicates that the improved structure meets the requirements. For the bending condition the stiffness of the whole body frame can be evaluated by bending deformation at the bottom of the frame. The maximum deformation at the left and right longitudinal beam of the frame bottom is $3.236 \mathrm{~mm}$ and $3.721 \mathrm{~mm}$ respectively, so the average deformation of two sides is $3.479 \mathrm{~mm}$. The bending stiffness value $K$ of body frame is $2.87 \times 10^{4} \mathrm{~N} / \mathrm{mm}$ after topology optimization, which is decreased slightly compared with the bending stiffness of the prototype, but still meets the design requirements of strength and stiffness. The relative deformation of doors and windows is decreased than the prototype, which indicates that the distribution of frame is more reasonable and coordinate after the topology optimization.

\section{Bending and Torsion Condition Stiffness Characteristics}

Analysis results chart of the primary position is shown in Fig. 11.

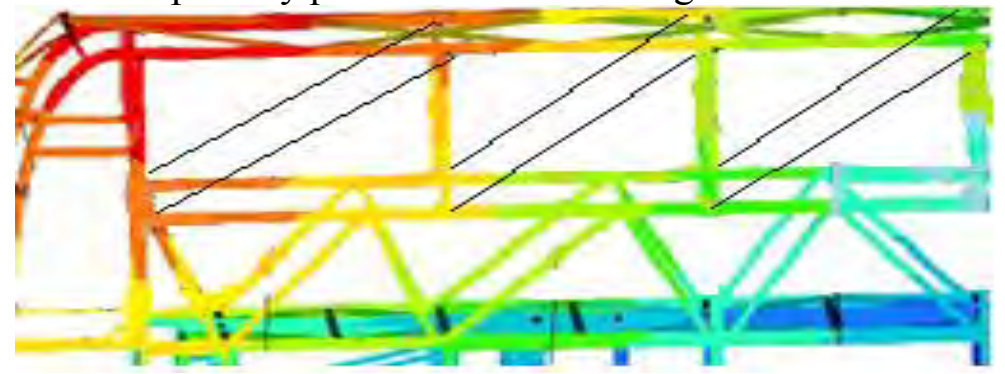

Fig. 11 Bending and Torsion Condition Measure Diagram 
The analysis results show that the largest deformation position is the front window, and the deformation value is $1.465 \mathrm{~mm}$, which illustrates that the local deformation of body is uniform. The torsion stiffness of the whole body frame can be evaluated by torsional angle value of both sides of the frame. In the post-processing software the torsion angle value $\theta$ can be gotten and it is $0.235^{\circ}$. The torsion stiffness value $K$ of the whole body frame is $1.87 \times 10^{4} \mathrm{Nm} / \mathrm{deg}$, which is decreased slightly compared with the torsional stiffness of prototype, but still within a reasonable range.

\section{Modal Characteristics}

Modal characteristics analysis is to verify the dynamic performance after optimization, and the ultimate purpose of the analysis is to prove the global performance of the mechanical systems. Therefore, the body frame's formation characteristics and the corresponding modal frequency values of the first to eighth orders are calculated in the finite element software, and the results before and after optimization are compared. The aim is to determine the optimization in a reasonable scope and ensure the effectiveness. Modal analysis is carried out by using finite element analysis software Radioss in the Altair Hyper-works. The body frame's performance comparison before and after optimization is shown in table 1 .

Tab. 1 the Body Frames Performance Comparison before and after Optimization

\begin{tabular}{cccc}
\hline Object & Before optimized & After optimized & $\begin{array}{c}\text { Change } \\
\text { value }\end{array}$ \\
\hline Mass $[\mathrm{Kg}]$ & 18690 & 18210 & 480 \\
The first order modal $[\mathrm{Hz}]$ & 7.926 & 9.457 & 1.531 \\
The second order modal $[\mathrm{Hz}]$ & 9.237 & 10.626 & 1.389 \\
The third order modal $[\mathrm{Hz}]$ & 14.806 & 15.924 & 1.118 \\
The fourth order modal $[\mathrm{Hz}]$ & 14.927 & 18.106 & 3.179 \\
The fifth order modal $[\mathrm{Hz}]$ & 17.223 & 20.233 & 3.010 \\
The sixth order modal $[\mathrm{Hz}]$ & 18.041 & 20.635 & 2.594 \\
The seventh order modal $[\mathrm{Hz}]$ & 18.723 & 21.826 & 3.103 \\
The eighth order modal $[\mathrm{Hz}]$ & 20.126 & 22.927 & 2.801 \\
\hline
\end{tabular}

Table 1 shows that the total mass of bus body frame has reduced $480 \mathrm{~kg}$ after optimization and each order modal is increased compared with the result before optimization in varying degrees. The modal analysis and calculation results show that the optimized body frame achieves the purpose of lightweight and dynamic performance is better than the original frame.

\section{Summary}

Topology optimization design space of bus body structure is established by Altair-OptiStruct software in this paper, and the performance of structure is obtained by static and dynamic analysis. The results are as follows:

1) Base on the prototype structure of body and optimized results, the new finite element model is established and the each order modal is greatly improved. Modal characteristics are reasonable.

2) Topology optimization of bus body structure is analyzed in order to lighten weight. Comparing with its quality before and after optimization, the conclusion indicates that the weight of frame have decreased by $3.8 \%$.

3) The results of optimization can not only accumulate experience of modern design for the structural design engineer, but also provide important reference for structure improvement and optimization design.

\section{Acknowledgement}

This research was supported by Discipline Foundation Project of Shanghai Dianji University (12XKJC02) and Research Project of Science and Technology Committee of Minhang District of Shanghai (2012MH152). 


\section{References}

[1]Jian Wang, Luzou Zhang, Geng-dong Cheng and Xu Guo, Optimal Topology Design of Truck Frame with Stress Constrains, Automotive Engineering. 1(1997)15-19.

[2]Zhi-lin Wu, Ming-xing Zhou, Guo-zhu Niu and Kun Liu, Structural Analysis and Optimization Design of TVM, International Conference on Mechanical Engineering and Mechanics. (2009)398-403.

[3]Ru-wen Chen, Analysis of the Topology Optimization, Electrical Information. 20(2004)42-44.

[4]Ya-jun Hong, Cheng Xu and Ya-ping Wang, Finite Element Analysis and Topology Optimization of a Shelter, International Conference on Mechanical Engineering and Mechanics.(2009)878-882.

[5] Yu Ma and Yu-wen Li, Fatigue Life Analysis of the Van Body Frame Based on ANSYS, IEEE Conference Publications. (2011)3980-3983.

[6]Tianze Huang, Bus Body Structure, Hunan University Press, Hunan, 1998.

[7]Kao, The Finite Element Method in Engineering, Pergamen Press, Oxford, 1982. 